\title{
Erratum to: Effects of Supplemental Feeding of Common Carp (Cyprinus carpio) with Iron Nanoparticles and Probiotic Lactobacillus on Blood Biochemical Factors
}

\author{
S. Ziaei-nejad ${ }^{a, *}$, N. Karami Abaei ${ }^{a}$, B. Nemat Doost ${ }^{a}$, and S. A. Johari ${ }^{b}$ \\ ${ }^{a}$ Department of Fisheries, Faculty of Natural Resources, Khatam Al-Anbia University of Technology, \\ Khuzestan, Behbahan, Iran \\ ${ }^{b}$ Department of Fisheries, Faculty of Natural Resources, University of Kurdistan, Sanandaj, Iran \\ *e-mail: zbsaeed@yahoo.com
}

DOI: $10.1134 /$ S1062359021060078

The affiliation of the fourth author (Seyed Ali Johari) should read as follows:

Department of Fisheries, Faculty of Natural Resources, University of Kurdistan, Sanandaj, Iran The original article can be found online at

https://doi.org/10.1134/S1062359021020163 